\title{
Yttrium-90 Microsphere Radioembolization
}

National Cancer Institute

\section{Source}

National Cancer Institute. Yttrium-90 Microsphere Radioembolization. NCI Thesaurus.

Code 1133735.

Antineoplastic treatment consisting of catheter injection of yttrium Y 90 microspheres.

When injected into the tumor vascular bed, yttrium Y 90 microspheres occlude tumor

blood vessels and deliver a cytotoxic dose of beta radiation to the tumor site, which may

result in tumor cell death and tumor regression. 